Artigo

\title{
Avaliação de um Modelo Estocástico de Erro Multidimensional Aplicado a Estimativas de Precipitação por Satélite
}

\author{
Aline Schneider Falck ${ }^{1}$, Daniel Vila ${ }^{1}$, Javier Tomasella ${ }^{2}$, Viviana Maggioni ${ }^{3}$, Fábio L.R. Diniz ${ }^{1}$ \\ ${ }^{1}$ Centro de Previsão de Tempo e Estudos Climáticos, Instituto Nacional de Pesquisas Espaciais, \\ São José dos Campos, SP, Brasil. \\ ${ }^{2}$ Centro Nacional de Monitoramento e Alerta de Desastres Naturais, \\ Cachoeira Paulista, SP, Brasil. \\ ${ }^{3}$ George Mason University, Fairfax, VA, Estados Unidos da América
}

Recebido: 7/3/2014 - Aceito: 16/5/2015

\begin{abstract}
Resumo
Uma das principais aplicações das estimativas de precipitação por satélite é a modelagem hidrológica em bacias onde a rede convencional e em tempo real de pluviômetros são precárias no que se refere à resolução espacial e temporal de dados. Neste trabalho discute-se o desempenho do modelo de erro de precipitação por satélite estocástico multidimensional - SREM2D (do inglês, Two-Dimensional Satellite Rainfall Error Model), o qual simula conjuntos de campos diários de precipitação com os mesmos padrões estatísticos (dispersão) que a diferença dos campos de chuva estimados por satélite e pluviômetro de uma série maior. A maioria dos modelos tratam o erro como uma medida uni-dimensional sem o reconhecimento que a precipitação é um processo intermitente no tempo e no espaço. O modelo SREM2D caracteriza a estrutura espacial, a dinâmica temporal e a variabilidade espacial do erro de estimativa das taxas de precipitação. Este trabalho avalia os resultados das simulações do SREM2D para diversos algoritmos de estimativa de precipitação por satélite na bacia dos rios Tocantins-Araguaia. Resultados mostram que o conjunto obtido através das realizações do modelo SREM2D reduziram o viés dos algoritmos de estimativa de precipitação por satélite principalmente para bacias com área de drenagem superior a $12.000 \mathrm{~km}^{2}$.
\end{abstract}

Palavras chave: modelo estocástico, métricas de calibração, estimativas de precipitação por satélite, modelagem hidrológica

\section{Evaluation of a Multidimensional Stochastic Error Model Applied to Satellite Rainfall Estimates}

\begin{abstract}
One of the most important applications of satellite rainfall estimates is the hydrological modeling in basins where the conventional and real time rain gauges networks are inadequate in term of the spatial and temporal resolution. This study discuss the performance of the multidimensional stochastic error model (SREM2D), which simulates an ensemble of daily precipitation fields with the same statistical patterns (spread) as the differences of satellite precipitation fields and rain gauges of a longer data series. Most models treat errors only in one dimension, without recognizing that rainfall is a time and space intermittent process. The SREMD2 model characterize the spatial and temporal structure, and the stail variability of errors, of rainfall estimates. This study assess SREM2D simulations results for several rainfall estimates algorithms in the Tocantins-Araguaia river basin. Results show that the ensemble derived from the SREM2D model reduced bias, of the satellite precipitation estimation algorithms mainly for basin with drainage area higher than $12000 \mathrm{~km}^{2}$.
\end{abstract}

Keywords : stochastic model, metrics calibration, satellite rainfall estimate, hydrological modeling .

Autor de correspondência: Aline Schneider Falck, aline.falck@cptec.inpe.br, asfalck@gmail.com. 


\section{Introdução}

A distribuição espacial e temporal da precipitação afeta uma infinidade de processos físicos e biológicos, estando entre eles a modulação do regime hidrológico das bacias hidrográficas. O funcionamento ecológico dos ecossistemas e atividades de grande impacto sócio-econômico também encontram-se relacionadas com o regime hidrológico.

No caso de regiões tropicais, os padrões de precipitação apresentam forte variação espacial e temporal, inclusive em uma escala inter-anual, manifestada na forma de episódios de secas e cheias (Marengo, 2004). Frequentemente as precipitações não tem duração maior do que algumas horas a cada evento, e são influenciadas por amplo espectro de fenômenos que variam desde a pequena até a grande escala. Devido a sua grande variabilidade espacial e temporal, a precipitação é uma das variáveis atmosféricas mais difíceis de se medir e estimar. Nesse contexto, a precipitação estimada por satélite apresenta-se como uma opção interessante uma vez que permite obter campos de precipitação com resolução espacial e temporal desejável na maioria das aplicações hidrológicas. Entretanto, essas estimativas possuem incertezas associadas aos tipos de sensores.

A técnica infravermelho térmico (IR, do inglês infrared) baseia-se na temperatura de topo frio das nuvens, com isto é possível avaliar a evolução dos sistemas precipitantes compostos por nuvens de grande desenvolvimento vertical. Além disso, possui alta resolução temporal, pois os sensores encontram-se a bordo de satélites geoestacionários (GEO, do inglês geostationary). No entanto, sua principal desvantagem é que estas estimativas podem ser contaminadas por nuvens do tipo cirrus que são frias e rasas superestimando a precipitação, além de ser uma medida indireta e empírica das taxas de precipitação associadas com estes sensores. A técnica micro-ondas passivas (PMW, do inglês passive microwave) fornece medida direta das taxas de precipitação sobre os oceanos, porém as desvantagens estão relacionadas com a baixa resolução espacial e temporal, além de fornecer medidas indiretas de taxas de precipitação sobre a área continental (espalhamento por gelo). No caso das micro-ondas ativas (AMW, do inglês active microwave) estas fornecem medidas diretas da estrutura vertical da precipitação, porém, as limitações existentes estão relacionadas a estreita faixa de dados obtidas por satélites de órbita baixa (LEO, do inglês low earth orbit), além da sensibilidade às taxas de precipitação. Em busca de melhorar as deficiências de cada método, a maioria dos algoritmos de estimativa de chuva por satélite optam por utilizar técnicas híbridas, utilizando as técnicas AMW e PMW associadas a dados do IR, além de correções com dados de superfície, com o intuito de minimizar estas incertezas.

Por isso, diversos estudos quantificaram as incertezas associadas nas estimativas de precipitação por satélite (co- mo por exemplo Huffman 1997, Tian et al., 2007, Sapiano and Arkin 2009, Anagnostou et al., 2010). Mais recentemente, estudos investigaram o erro de precipitação por satélite em escala de tempo relevantes e sua propagação através de modelos hidrológicos. Hong et al. (2006) quantificaram o erro aleatório na estimativa de precipitação por satélite sobre uma bacia no Mississipi, como uma função não-linear da escala de integração espaço-temporal da precipitação, intensidade da chuva e a frequência de amostragem, além de avaliar a influência do erro de estimativa de precipitação, em resposta a propagação do modelo hidrológico e concluíram que a quantificação de erro da precipitação como uma função não-linear da magnitude das chuvas em diferentes resoluções espaciais e temporais proporcionam uma quantificação mais realista do erro de estimativa de precipitação em relação ao erro de estimativa de precipitação convencional como proporção fixa das taxas de chuva. Hossain e Anagnostou (2006) argumentam que, devido a incerteza na delimitação das áreas de chuva e não-chuva, é necessária a formulação de modelos de erro de precipitação para escalas de tempo e espaço hidrologicamente relevantes.

A maioria dos modelos tratam o erro como uma medida uni-dimensional sem o reconhecimento que a precipitação é um processo intermitente no tempo e no espaço. O modelo de erro de precipitação por satélite estocástico multidimensional - SREM2D (do inglês, Two-Dimensional Satellite Rainfall Error Model) caracteriza a estrutura espacial da delimitação de áreas de chuva e não-chuva, dinâmica temporal do viés das estimativas de precipitação e a variabilidade espacial do erro de estimativa das taxas de precipitação, por meio de nove métricas de calibração Este modelo de erro possui melhor desempenho no que diz respeito a preservação da estrutura de erro das escalas temporal e espacial da variável precipitação. O objetivo deste estudo é avaliar como prova de conceito, o desempenho do modelo de erro SREM2D, em reproduzir conjuntos de estimativa de precipitação por satélite com maior precisão na bacia dos rios Tocantins-Araguaia.

Para atingir os objetivos descritos nesta seção, o estudo foi organizado da seguinte maneira: na seção dois estão descritos os dados e a metodologia de implementação do modelo estocástico SREM2D, a seção três apresenta a discussão dos resultados obtidos e a seção quatro trata das conclusões e trabalhos futuros.

\section{Dados e Metodologia}

A bacia hidrográfica dos rios Tocantins-Araguaia fica situada em uma importante região hidroclimática, influenciada por diferentes sistemas que atuam na região de estudo, entre eles: a zona de convergência intertropical, os sistemas frontais, as linhas de instabilidade e a zona de convergência do oceano Atlântico Sul (Marengo e Nobre, 2009). Bacia de grande porte, possui área de drenagem de aproximadamente $764.000 \mathrm{~km}^{2}$, ocupa cerca de $9 \%$ do 
território nacional, abrangendo o estado do Tocantins e parte dos estados de Goiás, Mato Grosso e Maranhão, suas coordenadas geográficas estão compreendidas entre $2^{\circ} \mathrm{e}$ $18^{\circ} \mathrm{S}$ e $46^{\circ}$ e $56^{\circ} \mathrm{W}$ (Figura 1 ).

Este estudo utiliza dados acumulados diários obtidos a partir de pluviômetros convencionais e automáticos e dados de estimativa de precipitação por satélite dos algoritmos CMORPH (Joyce et al., 2004), 3B42RT (Huffman et al., 2007), HYDROE (versão aprimorada do autoestimador desenvolvido por Vicente et al., 1998) e GSMaP (Aonashi et al., 2009), compreendendo as estações chuvosas (2008/2009 e 2009/2010) e secas (2009 e 2010). As estações chuvosas referem-se aos períodos 15/10/2008$14 / 05 / 2009$ e 15/10/2009-14/05/2010, e as estações secas referem-se aos períodos 15/05/2008-14/10/2009 e 15/05/2009-14/10/2010.

Os produtos de estimativa de chuva por satélite foram agregados para resolução temporal diária e resolução espacial de $0,25^{\circ}$ x $0,25^{\circ}$ de grade, substituindo suas resoluções nativas quando necessário. Os dados pluviométricos foram interpolados através da técnica de optimal interpolation (Gandin, 1959). Logo, os pixels que estão ao redor dos pluviômetros até a distância máxima de $25 \mathrm{~km}$ de raio (pixels contíguos) são considerados para a análise enquanto que aqueles que ficam numa distância maior são desconsiderados para evitar as incertezas geradas pelo processo de interpolação.

Dados pluviométricos de aproximadamente 300 estações foram utilizadas neste estudo, cuja distribuição espacial está representada na Figura 1. Estes dados foram obtidos junto ao banco de dados do Instituto Nacional de Pesquisas Espaciais (INPE), sendo o controle de qualidade realizado pelo próprio centro.

\subsection{Implementação do modelo de erro estocástico multidimensional (SREM2D)}

O modelo SREM2D ("Two Dimensional Satellite Rainfall Error Model") foi desenvolvido por Hossain e

\section{Legenda}
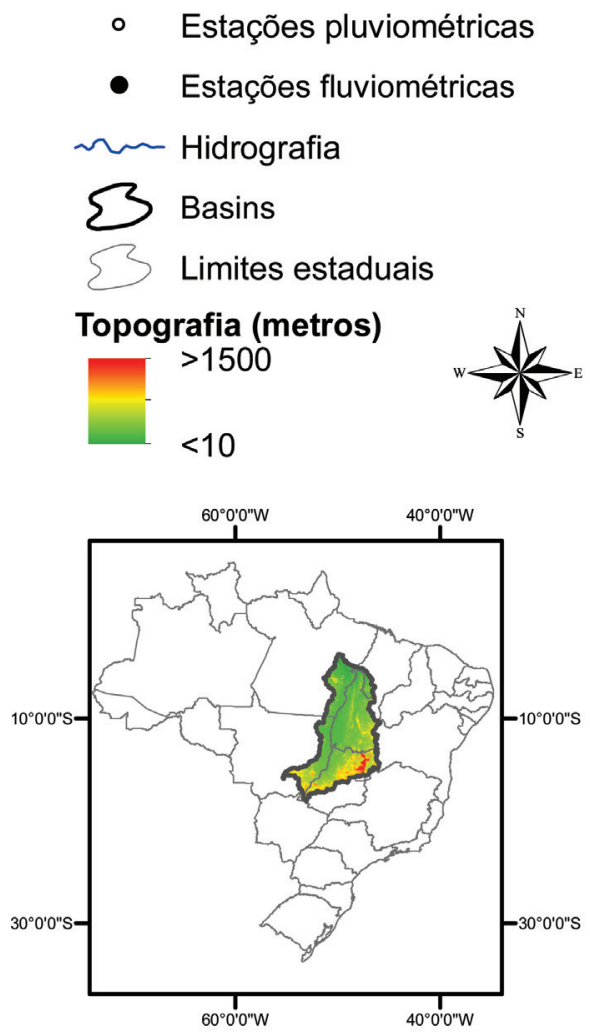

Base de dados Hidrografia - ANA, 2010 Limites estaduais: IBGE, 2007 Estações: Banco de dados INPE Datum: WGS 84

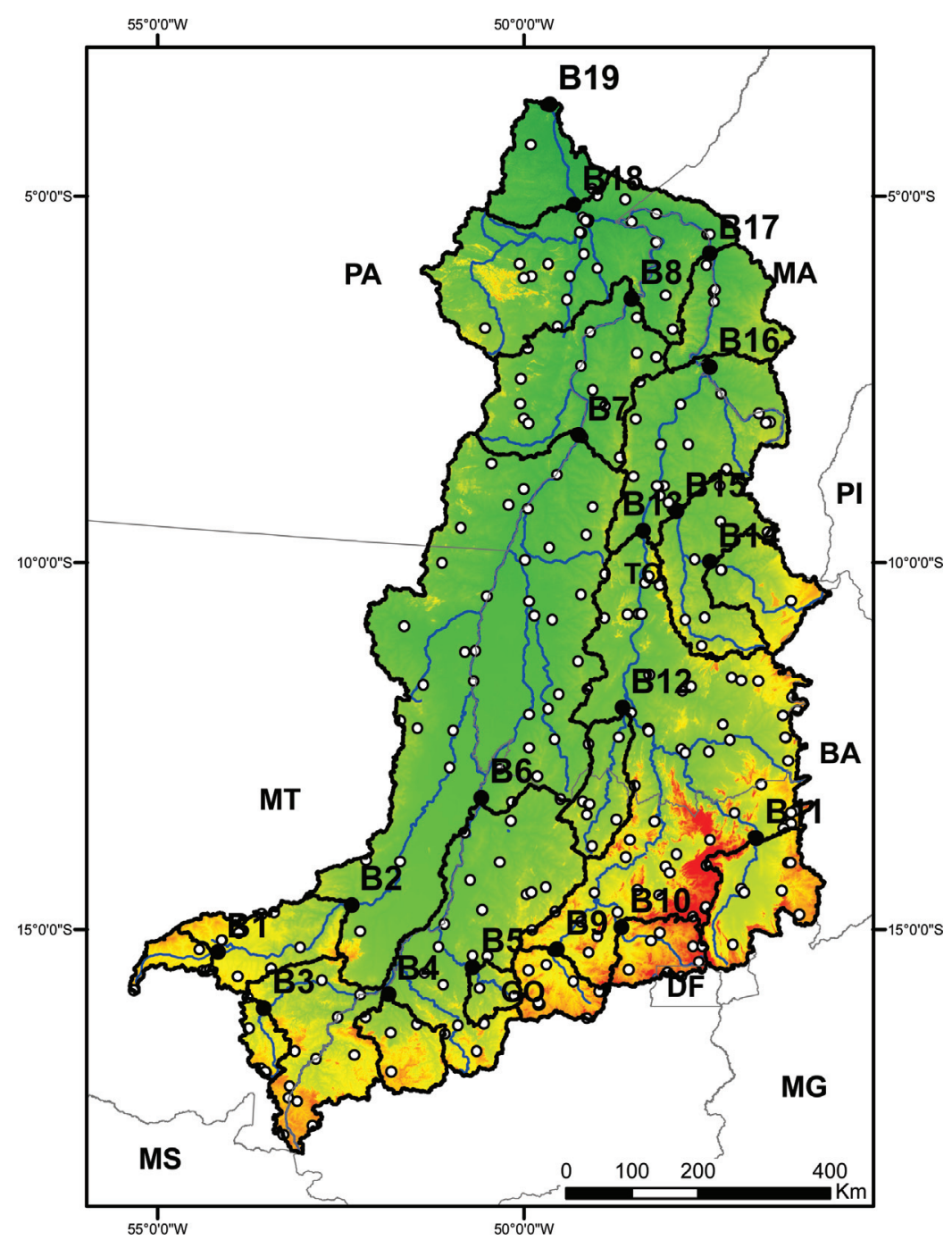

Figura 1 - Localização da bacia hidrográfica dos rios Tocantins-Araguaia em território brasileiro e porções estaduais pertencentes, hidrografia dos principais rios, elevação (metros) e distribuição das estações pluviométricas situadas na bacia dos rios Tocantins-Araguaia. 
Anagnostou (2006) para simular conjuntos de precipitação por satélite baseados em campos de chuva de maior precisão chamados de "referência", sendo o principal objetivo caracterizar a estrutura multidimensional estocástica do erro das estimativas de precipitação por satélite como função de escala. Neste estudo, a implementação do modelo de erro utiliza a definição de entrada SREM2D inversa, ou seja, a estrutura multidimensional dos desvios em relação à precipitção de referência (isto é, precipitação pluviométrica) é derivada com relação às estimativas de precipitação por satélite para gerar conjuntos de campos de precipitação semelhantes ao de referência para cada produto de satélite.

\subsection{Cálculo das métricas de calibração SREM2D}

Para o cálculo das métricas de erro SREM2D, considera-se que a "referência" são os dados pluviométricos e o "sensor" são dados de estimativa de precipitação por satélite dos diversos algoritmos mencionados. A metodologia das métricas de calibração descritas abaixo foram retiradas do trabalho de Hossain et al. (2009). As estatísticas básicas podem ser encontradas em Wilks (1995). Maiores informações sobre o modelo SREM2D podem ser encontradas em Hossain e Anagnostou (2006). As métricas 1, 2, 3 e 4 consideram a tabela de contingência representada na Figura 2. Coletivamente, as nove métricas abaixo representam a estrutura de erro multidimensional dos algoritmos de estimativa de precipitação por satélite.

a. Probabilidade de detecção de chuva (Métrica 1)

A probabilidade de detecção de chuva $\left(\mathrm{POD}_{\text {rain }}\right)$ é calculada pela Eq. (1).

$$
\operatorname{POD}_{\text {rain }}=\frac{\mathrm{N}_{\mathrm{A}}}{\left(\mathrm{N}_{\mathrm{A}}+\mathrm{N}_{\mathrm{C}}\right)}
$$

O SREM2D modela a dependência da POD $_{\text {rain }}$ com taxa de precipitação através do modelo de regressão logística (Eq. (2)), sendo os parâmetros de ajuste A e B as métricas utilizadas pelo SREM2D.

$$
\mathrm{POD}_{\text {rain }}(\mathrm{REF})=\frac{1}{\mathrm{~A}+\exp \left(-\mathrm{BR}_{\mathrm{REF}}\right)}
$$

\section{b. Probabilidade de detecção de não-chuva (Métrica 2)}

A Probabilidade de detecção de não-chuva $\left(\mathrm{POD}_{\text {norain }}\right)$ é calculada pela Eq. (3), considerando a tabela de contingência ilustrada na Figura 2. A POD norain é uma probabilidade unitária onde a referência e o sensor apresentam valores de chuva iguais a zero, ou seja, o satélite detecta o processo sem chuva.

$$
\mathrm{POD}_{\text {norain }}=\frac{\mathrm{N}_{\mathrm{D}}}{\left(\mathrm{N}_{\mathrm{B}}+\mathrm{N}_{\mathrm{D}}\right)}
$$

c. Distribuição das taxas de chuva de falso alarme (Métrica 3)

A função densidade de probabilidade $\left(\mathrm{D}_{\text {false }}\right)$ é definida de modo a caracterizar a distribuição de probabilidade das estimativas de chuva por satélite das taxas de chuva de falso alarme. A $\mathrm{D}_{\text {false }}$ é calculada de acordo com a Eq. (4). De acordo com Hossain e Anagnostou (2006) a distribuição de probabilidade tende aparecer exponencial e desta forma o primeiro e segundo momento podem ser definidos utilizando apenas o parâmetro $\lambda$ da distribuição, cujo o valor inverso $(1 / \lambda)$ é a métrica utilizada pelo SREM2D

$$
\mathrm{D}_{\text {false }}=\frac{\mathrm{N}_{\mathrm{B}}}{\left(\mathrm{N}_{\mathrm{A}}+\mathrm{N}_{\mathrm{B}}\right)}
$$

\section{d. Comprimento de correlação (Métricas 4, 5 e 8)}

Para identificar o comprimento de correlação de erro de detecção de chuva - $\mathrm{CL}_{\text {rain }}$ e não-chuva - $\mathrm{CL}_{\text {norain }}$, assume-se uma função exponencial simples de auto-covariância no SREM2D, na qual o comprimento de correlação é a distância de separação $1 / \mathrm{e}=0,3678$. Sendo que, para identificar o comprimento de correlação de áreas de chuva $\mathrm{CL}_{\text {rain }}$ e não-chuva - $\mathrm{CL}_{\text {norain }}$, atribuí-se o valor 1,0 e 0,0 , respectivamente.

Para identificar o comprimento de correlação da chuva condicional - $\mathrm{CL}_{\text {ret }}$, considera-se os acertos de chuva do satélite e pluviômetro (os dois valores positivos) e na sequência calcula-se o comprimento de correlação em termos do erro de recuperação definido como a diferença logarítmica entre o dado de referência e a estimativa de satélite.

e. Distribuição das taxas de chuva condicionais (Métricas 6 e 7)

O condicional não nulo de taxas de chuva de referência $\left(R_{R E F}\right)$ está, estatisticamente relacionado no SREM2D à correspondente condição de taxa de chuva por satélite $\left(\mathrm{R}_{\mathrm{SAT}}\right)$, como:

$$
\mathrm{R}_{\mathrm{REF}}=R_{\mathrm{SAT}} \varepsilon_{\mathrm{s}}
$$

onde o parâmetro de erro de recuperação do satélite, $\varepsilon_{\mathrm{s}}$, é assumido como sendo uma distribuição log-normal. A vantagem de tal pressuposto é que uma transformação logarítmica $\left[\log \left(\mathrm{R}_{\mathrm{REF}}\right)-\log \left(\mathrm{R}_{\mathrm{SAT}}\right)\right]$ permite $\varepsilon_{\mathrm{s}}$, de forma a ser mapeado pelo desvio de uma Gaussiana $\mathrm{N}(\mu, \sigma)$, onde $\mu \mathrm{e}$ $\sigma$ são a média e desvio padrão, respectivamente.

\section{f. Correlação temporal Lag-1 (Métrica 9)}

O parâmetro do erro de recuperação $\varepsilon$ é auto-correlacionado espacialmente e temporalmente e, essa estrutura de tempo-espaço é considerada no SREM2D. Para a correlação temporal, uma função auto-regressiva é utilizada para identificar a variabilidade temporal de $\mu$.

O diagrama lógico do modelo SREM2D ilustrado na Figura 2 apresenta as nove métricas de calibração enumeradas de 1 a 9, bem como apresentadas anteriormente (itens a-f). Após a obtenção das métricas de calibração do modelo SREM2D, as mesmas foram utilizadas como parâmetros de entrada nesse modelo para corrigir o erro das estimativas de precipitação por satélites com relação à referência utilizada. Para isso o modelo utiliza o método de Monte Carlo 

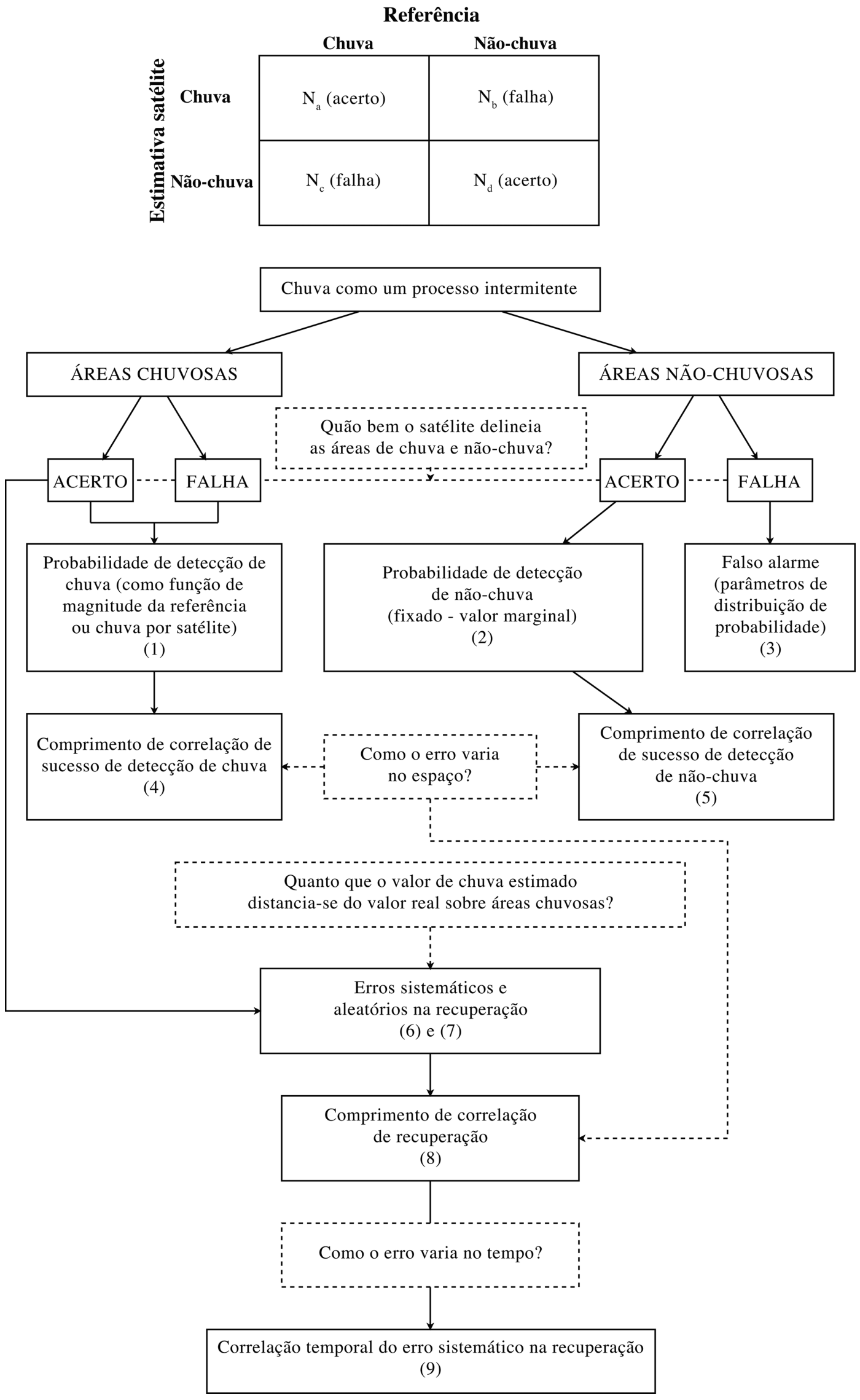

Figura 2 - Diagrama lógico conceitual das métricas de erro SREM2D. FONTE: Adaptado de Hossain e Huffman (2008). 
corrigindo os campos estimados por satélite com base em dados de maior precisão (isto é, dados de referência neste caso pluviômetros). Neste experimento optou-se por 100 iterações, ou seja, conjuntos compostos por 100 membros.

\section{Resultados}

\subsection{Calibração do modelo SREM2D}

A Figura 3 mostra a probabilidade de detecção de chuva $\left(\mathrm{POD}_{\text {rain }}\right)$ em função da magnitude da precipitação, onde observam-se os menores valores de $\mathrm{POD}_{\text {rain }}$ para as menores taxas de precipitação diária, e os maiores valores próximos de 1 para chuvas intensas nas estações chuvosas e secas. Isto ocorre devido à dificuldade do satélite em detectar chuva estratiforme (onde efetivamente esta chovendo) e conseguir detectar mais facilmente chuvas convectivas. Além disso, observa-se que os valores de probabilidade de detecção de chuva são inferiores para as estações secas, devido ao menor número de dias com chuva para o período. Analisando o comportamento de cada algoritmo é possível observar que o HYDROE tem performance inferior durante

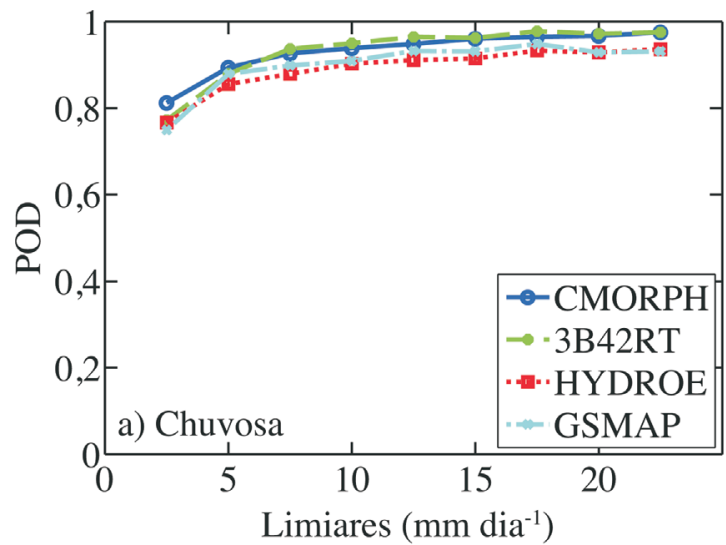

a estação chuvosa para todas as taxas de precipitação, enquanto o CMORPH e o 3B42RT possuem melhor desempenho. Ao contrário, nas estações secas o HYDROE possui melhor desempenho para as taxas de precipitação menores que $10 \mathrm{~mm} /$ dia.

A probabilidade de detecção de não-chuva ( POD $\left._{\text {norain }}\right)$ é ilustrada na Figura 4 , onde observa-se menores valores de $\mathrm{POD}_{\text {norain }}$ nas estações chuvosas para todos os algoritmos (da ordem de 60\%) do que nas estações secas (valores em torno de 90\%). Esse resultado é devido a maior probabilidade dos algoritmos acertarem a previsão de não chuva nas estações secas do que nas estações chuvosas.

A distribuição de probabilidade das taxas de chuva diária de falso alarme $\left(\mathrm{D}_{\text {false }}\right)$ é observada na Figura 5, onde os maiores valores de $\mathrm{D}_{\text {false }}$ correspondem às menores taxas de chuva diária, ou seja, o satélite detecta chuva que não é observada em superfície. A partir da análise desta figura, o comportamento deste parâmetro estatístico não mostra grande diferença para todos os algoritmos analisados e para todas as taxas de precipitação, tanto para as estações chuvosas quanto para as estações secas.

Figura 3 - Probabilidade de deteç̧ão de chuva para os algoritmos de estimativa de precipitação por satélite CMORPH, 3B42RT, HYDROE e GSMaP para as estações chuvosas (2008/2009 e 2009/2010) e secas (2009 e 2010) sobre a bacia dos rios Tocantins-Araguaia (esquerda e direita, respectivamente).


Figura 4 - Probabilidade de detecção de não-chuva dos algoritmos de estimativa de precipitação por satélite CMORPH, 3B42RT, HYDROE e GSMaP para as estações chuvosas (2008/2009 e 2009/2010) e secas (2009 e 2010) sobre a bacia dos rios Tocantins-Araguaia (esquerda e direita, respectivamente). 

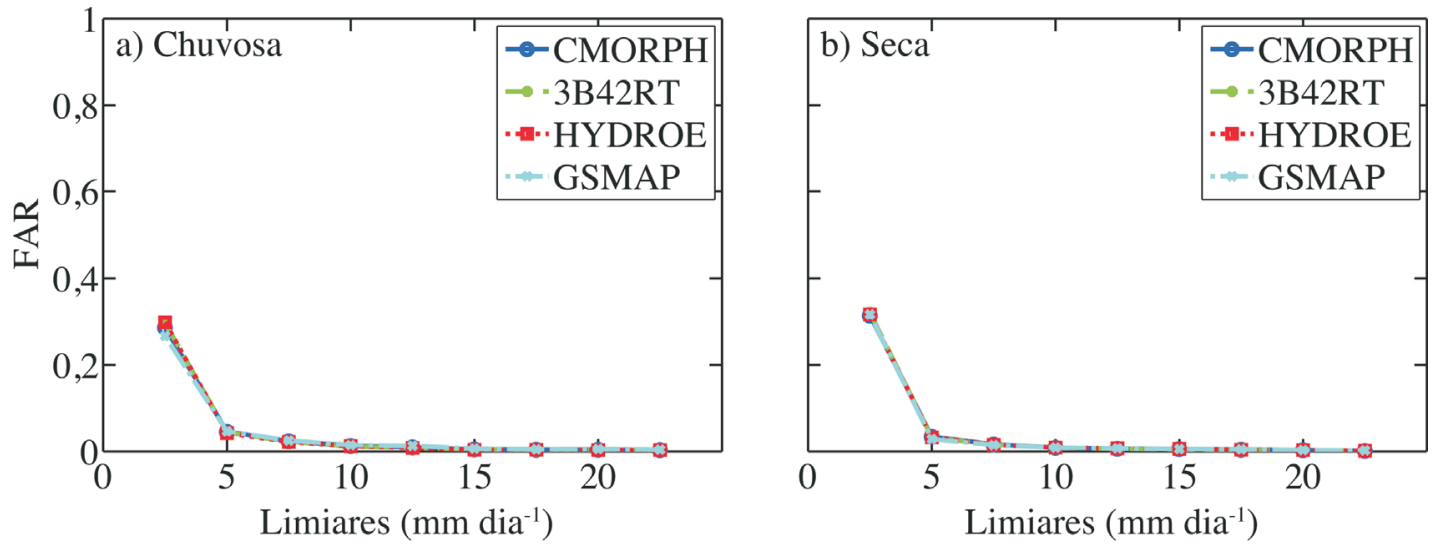

Figura 5 - Distribuição das taxas de chuva de falso alarme, para os algoritmos de estimativa de precipitação por satélite CMORPH, 3B42RT, HYDROE e GSMaP para as estações chuvosas (2008/2009 e 2009/2010) e secas (2009 e 2010) sobre a bacia dos rios Tocantins-Araguaia, (esquerda e direita, respectivamente).

A estrutura de covariância espacial de detecção de chuva, detecção de não-chuva e de recuperação de chuva (chuva condicional), são apresentadas nas Figuras 6, 7 e 8, respectivamente. A partir desses gráficos é possível dizer que o modelo exponencial é representativo da correlação espacial da detecção de chuva, não-chuva e da chuva condicional. Considera-se o valor de correlação igual a 1/e como o limiar para definir a distância máxima que dois pontos estão correlacionados entre si. Esses limiares para as estações chuvosas e secas são apresentados na Tabela 1. No
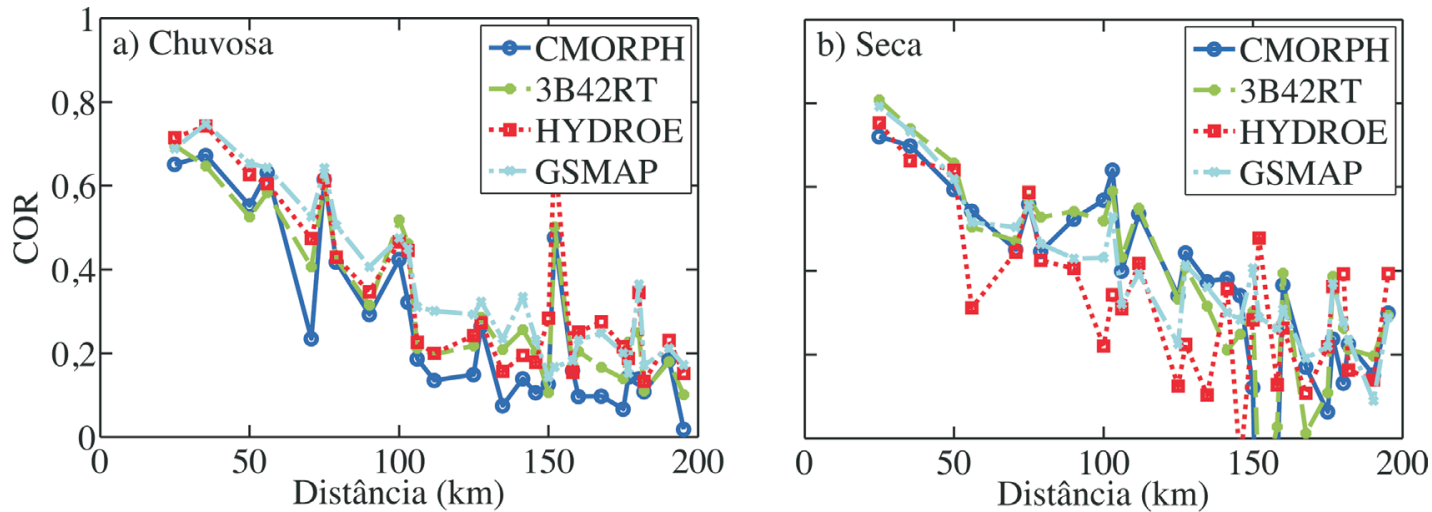

Figura 6 - Estrutura de covariância espacial de detecção de chuva, para os algoritmos de estimativa de precipitação por satélite CMORPH, 3B42RT, HYDROE e GSMaP comparado com dados observados para as estações chuvosas (2008/2009 e 2009/2010) e secas (2009 e 2010) sobre a bacia dos rios Tocantins-Araguaia (esquerda e direita, respectivamente).
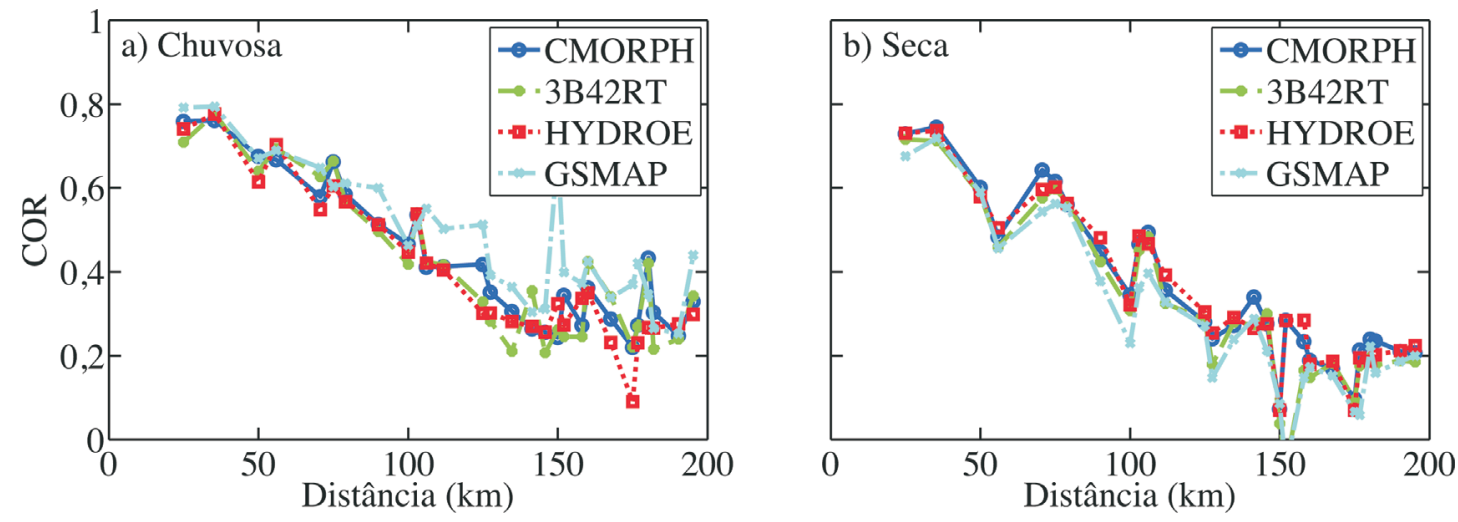

Figura 7 - Estrutura de covariância espacial de detecção de não-chuva, para os algoritmos de estimativa de precipitação por satélite CMORPH, 3B42RT, HYDROE e GSMaP comparado com dados observados para as estações chuvosas (2008/2009 e 2009/2010) e secas (2009 e 2010) sobre a bacia dos rios Tocantins-Araguaia (esquerda e direita, respectivamente). 

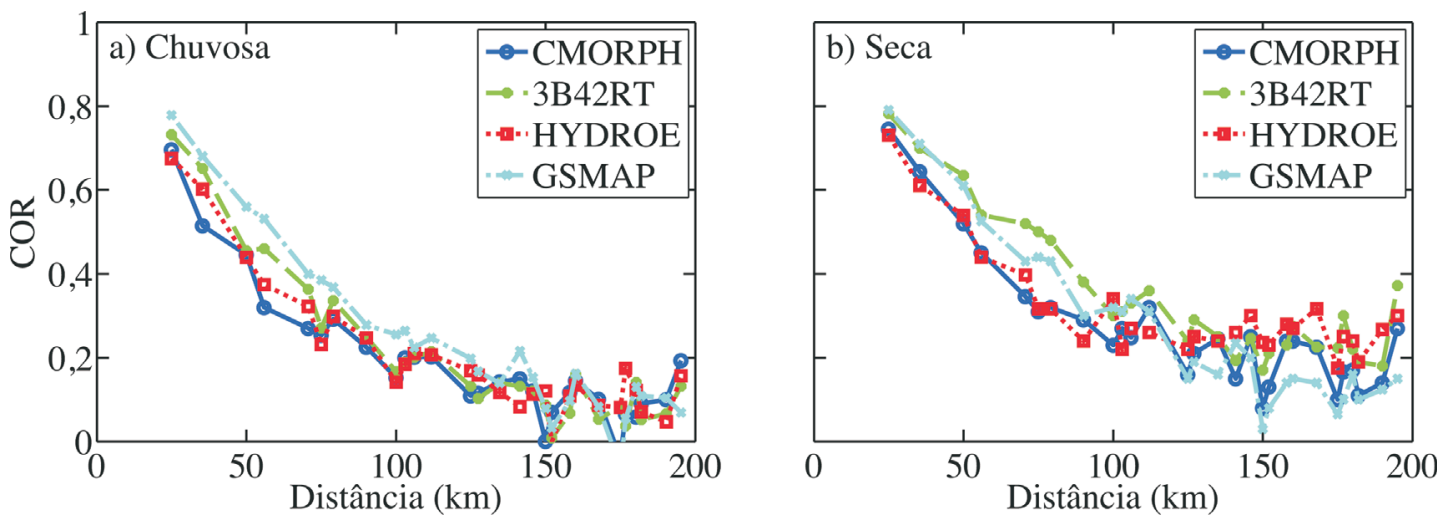

Figura 8 - Estrutura de covariância espacial de recuperação de chuva, para os algoritmos de estimativa de precipitação por satélite CMORPH, 3B42RT, HYDROE e GSMaP comparado com dados observados para as estações chuvosas (2008/2009 e 2009/2010) e secas (2009 e 2010) sobre a bacia dos rios Tocantins-Araguaia (esquerda e direita, respectivamente).

Tabela 1 - Métricas de calibração do modelo SREM2D para os algoritmos CMORPH, 3B42RT, HYDROE e GSMaP nas estações chuvosas (2008/2009 e 2009/2010) e secas (2009 e 2010) sobre a bacia dos rios Tocantins-Araguaia.

\begin{tabular}{|c|c|c|c|c|c|c|c|c|}
\hline \multirow[t]{2}{*}{ Metricas } & \multicolumn{2}{|c|}{ CMORPH } & \multicolumn{2}{|c|}{ 3B42RT } & \multicolumn{2}{|c|}{ HYDROE } & \multicolumn{2}{|c|}{ GSMaP } \\
\hline & Chuv & Seca & Chuv & Seca & Chuv & Seca & Chuv & Seca \\
\hline $\mathrm{POD}_{\text {rain }}$ parâmetro $\mathrm{A}$ & 0,07 & 0,17 & 0,09 & 0,17 & 0,07 & 0,08 & 0,08 & 0,15 \\
\hline $\mathrm{POD}_{\text {rain }}$ parâmetro $\mathrm{B}$ & 0,77 & 0,39 & 0,73 & 0,40 & 0,72 & 0,57 & 0,72 & 0,39 \\
\hline Falso alarme $(1 / \lambda)$ & 1,48 & 1,22 & 1,38 & 1,22 & 1,35 & 1,21 & 1,76 & 1,18 \\
\hline Média gauss. erro log. & $-0,19$ & $-0,23$ & $-0,06$ & $-0,06$ & $-0,32$ & $-0,10$ & 0,16 & 0,18 \\
\hline Des. Pad. gauss. erro log. & 1,40 & 1,44 & 1,28 & 1,42 & 1,49 & 1,51 & 1,50 & 1,52 \\
\hline $\mathrm{CL}_{\text {rain }}(\mathrm{km})$ & 65 & 125 & 85 & 125 & 85 & 55 & 105 & 110 \\
\hline $\mathrm{CL}_{\text {norain }}(\mathrm{km})$ & 130 & 100 & 120 & 100 & 120 & 100 & 140 & 90 \\
\hline $\mathrm{CL}_{\text {ret }}(\mathrm{km})$ & 55 & 100 & 75 & 75 & 65 & 75 & 85 & 90 \\
\hline Corr. temporal Lag-1 & 0,17 & $-0,12$ & 0,26 & $-0,05$ & 0,21 & 0,12 & 0,11 & 0,10 \\
\hline $\mathrm{POD}_{\text {norain }}(\%)$ & 0,55 & 0,88 & 0,58 & 0,89 & 0,61 & 0,89 & 0,61 & 0,91 \\
\hline
\end{tabular}

entanto, observa-se os menores comprimentos de correlação de chuva $\left(\mathrm{CL}_{\text {rain }}\right)$ para as estações chuvosas e maior $\mathrm{CL}_{\text {rain }}$ para as estações secas, exceto para o algoritmo HYDROE. Para a correlação de não chuva $\left(\mathrm{CL}_{\text {norain }}\right)$, observa-se os menores valores de $\mathrm{CL}_{\text {norain }}$ para as estações secas, na ordem de aproximadamente $100 \mathrm{~km}$ para todos os algoritmos, enquanto os valores $\mathrm{CL}_{\text {norain }}$ para as estações chuvosas são superiores a $120 \mathrm{~km}$. Para os comprimentos de correlação de recuperação de chuva $\left(\mathrm{CL}_{r e t}\right)$, observa-se menores valores de $\mathrm{CL}_{\text {ret }}$ para as estações chuvosas e maiores valores para as estações secas.

Enquanto para a correlação temporal, os valores do lag-1 (parâmetro de entrada do SREM2D) apresentam valores absolutos menores que 0.3 na estação chuvosa e menores que 0.15 na estação seca. Esses valores indicam a baixa correlação temporal diária que existe entre dois dias consecutivos, sugerindo a natureza convectiva dos sistemas atuantes na bacia dos rios Tocantins-Araguaia.

A média do erro logarítmico, $\mathrm{e}_{\mathrm{S}}$, pode ser observada na Figura 9. Pode-se neste caso ressaltar que o algoritmo de estimativa de chuva GSMaP subestima a chuva (valor posi- tivo) e os demais algoritmos superestimam a chuva (valor negativo) de acordo com a definição apresentada no item 2.2.e., tanto nas estações chuvosas quanto nas estações secas. No caso do algoritmo 3B42RT observou-se a menor média do erro logarítmico (em valor absoluto) nas duas estações, no qual é possível concluir que esse algoritmo representa melhor os dados de precipitação observada, enquanto o HYDROE apresentou o maior erro médio (em valor absoluto) na estação chuvosa.

A Figura 10 mostra o desvio padrão do erro logarítmico $(\sigma)$ para a estação chuvosa (esquerda) e a estação seca (direita). Neste caso, o menor valor de $\sigma$ esta relacionado com o algoritmo 3B42RT para as duas estações, em quanto as técnicas GSMPA e HYDROE têm os maiores valores tanto para a estação seca como para a estação chuvosa.

A Tabela 1 sumariza os valores obtidos para cada uma das nove métricas de calibração (parâmetros A e B, probabilidade de detecção de não-chuva, distribuição das taxas de falso alarme, comprimento de correlação de chuva, não-chuva e de recuperação, correlação temporal, distribui- 

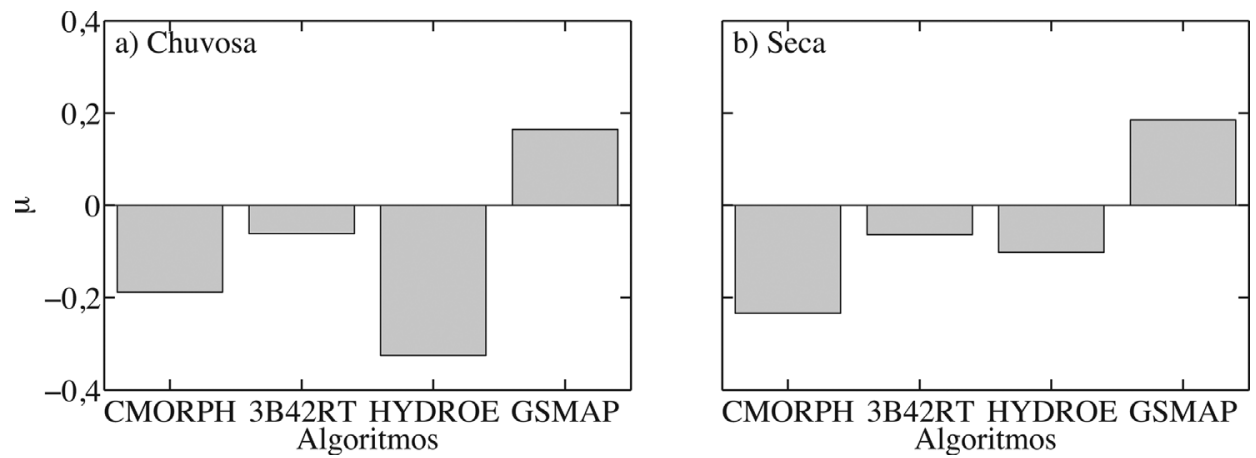

Figura 9 - Média do erro logarítmico SREM2D para os produtos de estimativa de precipitação por satélite CMORPH, 3B42RT, HYDROE e GSMaP nas estações chuvosas (2008/2009 e 2009/2010) e secas (2009 e 2010) sobre a bacia dos rios Tocantins-Araguaia (esquerda e direita, respectivamente).
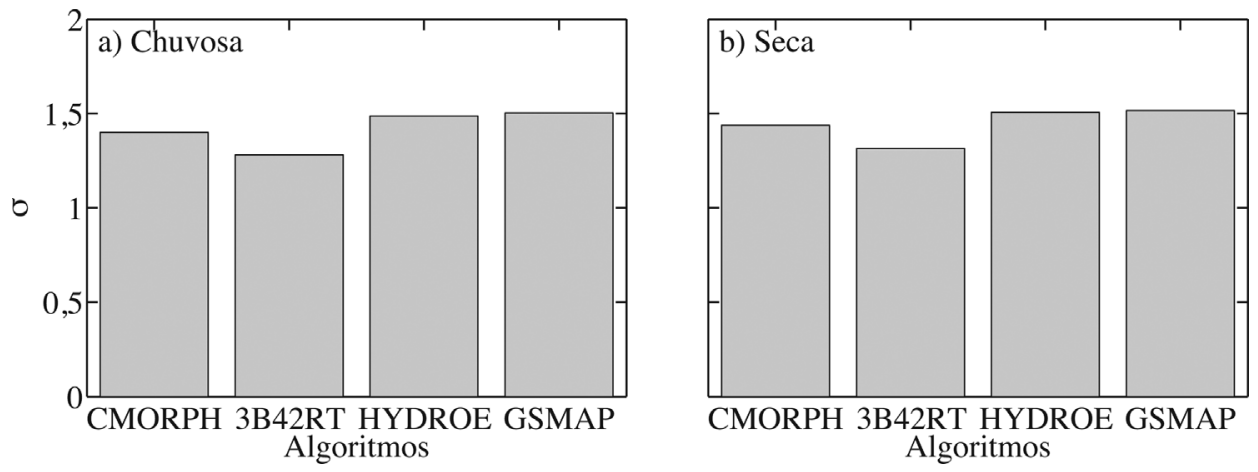

Figura 10 - Desvio padrão do erro logarítmico SREM2D para os produtos de estimativa de precipitação por satélite CMORPH, 3B42RT, HYDROE e GSMaP nas estações chuvosas (2008/2009 e 2009/2010) e secas (2009 e 2010) sobre a bacia dos rios Tocantins-Araguaia (esquerda e direita, respectivamente).

ção das taxas de chuva $\mu$ e $\sigma$ ) do modelo SREM2D, para os produtos de estimativa de precipitação por satélite CMORPH, 3B42RT, HYDROE e GSMaP aplicados sobre bacia hidrográfica dos rios Tocantins-Araguaia para os períodos chuvosos e secos, quando comparados com os valores obtidos nos estudos de Hossain et al. (2009).

\subsection{Avaliação do modelo SREM2D}

A Figura 11 mostra os hietogramas acumulados das estimativas de precipitação satélite com relação aos dados observados. O CMORPH e o HYDROE superestimam a precipitação observada, enquanto o GSMaP subestima a precipitação observada para todo o período. $\mathrm{O}$ algoritmo 3B42RT fica muito próximo ao dado de referência.

Nesta figura também avaliou-se os conjuntos de precipitação (100 membros) simulados pelo modelo SREM2D para cada um dos algoritmos analisados. Em todos os casos, a média do conjunto tende a se aproximar dos dados de precipitação observada (referência). Observa-se também que tanto para as estações chuvosas quanto para as estações secas, os dados de referência encontram-se envolvidos pelos conjuntos simulados pelo modelo SREM2D.

A partir desta análise, é possível concluir que o uso direto das estimativas de precipitação por satélite (com exceção do algoritmo 3B42RT) não reproduzem de forma correta o comportamento da precipitação observada. A partir da aplicação do modelo estocástico foi possível reproduzir padrões bem definidos e semelhantes aos dados de referência, evidenciando uma substancial melhoria do campo original estimado por satélite.

A quantificação do erro das estimativas por satélite podem ser observadas na Figura 12, através do viés relativo da precipitação (rBIAS), definido pela seguinte expressão:

$$
\operatorname{rBIAS}=\frac{\frac{1}{\mathrm{~N}} \sum_{\mathrm{k}=1}^{\mathrm{N}}\left(\hat{\mathrm{R}}_{\mathrm{k}}-\mathrm{R}_{\mathrm{k}}\right)}{\frac{1}{\mathrm{~N}} \sum_{\mathrm{k}=1}^{\mathrm{N}}\left(\hat{\mathrm{R}}_{\mathrm{k}}\right)}
$$

onde $\mathrm{N}$ é o número total de passos de tempo (dias), $\mathrm{R}$ é a precipitação observada, $\hat{R}$ é a estimativa da precipitação por satélite. Esta estatística foi calculado para cada um dos membros do conjunto derivado do modelo SREM2D.

Este parâmetro estatístico mede a diferença entre a precipitação observada por pluviômetros e as estimativas de precipitação por satélite (pontos na Figura 12) e o conjunto obtido pelo modelo SREM2D (box-plot na mesma figura), normalizada pela precipitação observada. Este parâmetro foi calculado para todas as sub-bacias do Tocan- 


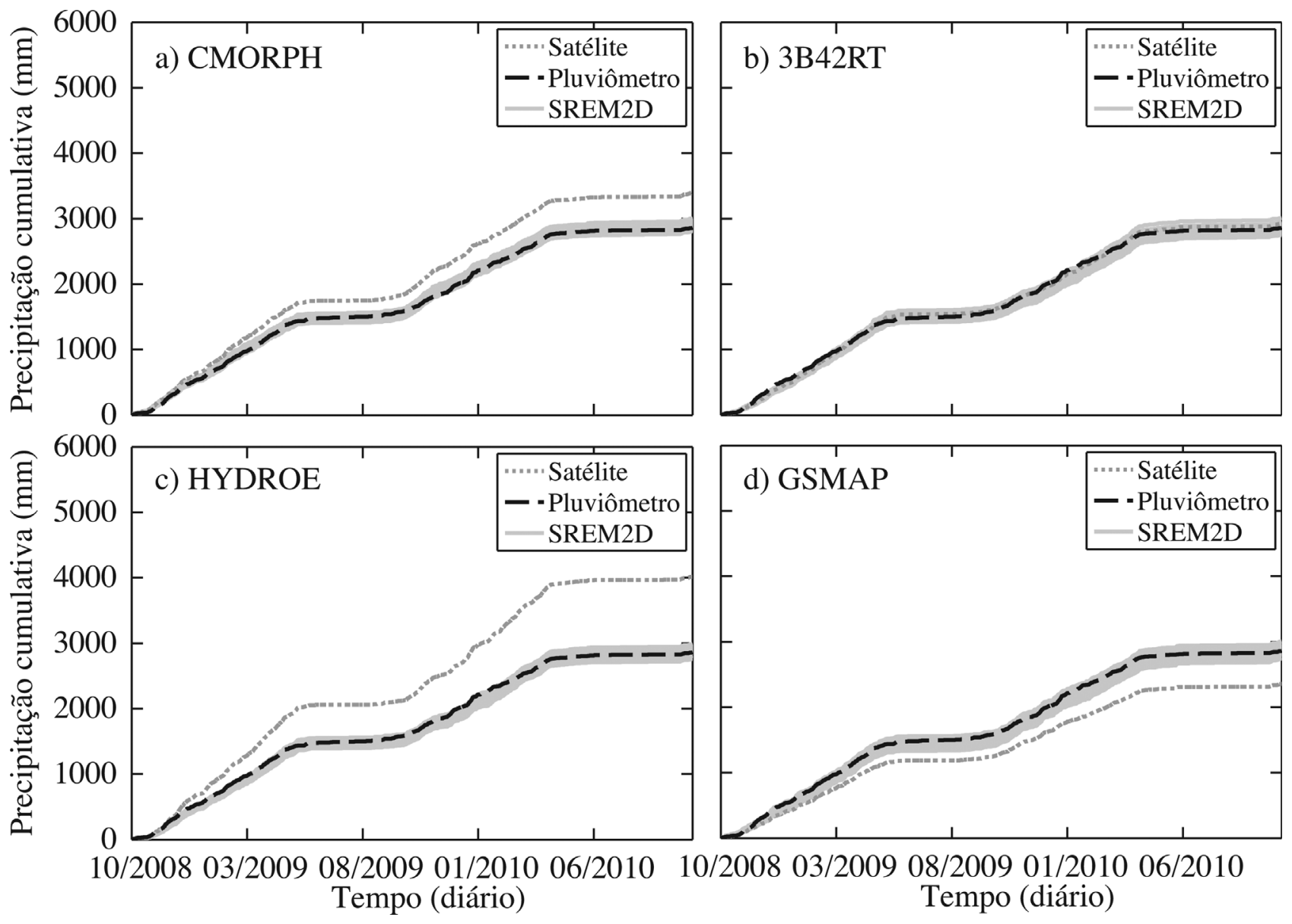

Figura 11 - Hietogramas acumulado para um conjunto de 100 realizações (linhas cinza médio), simulado pelo SREM2D e comparado com os dados de precipitação observada (linha cinza escura) e estimada por satélite (linha cinza claro) pelos algoritmos CMORPH (superior-esquerdo), 3B42RT (superior-direito), HYDROE (inferior-esquerdo) e GSMaP (inferior-direito), nas estações chuvosas (2008/2009 e 2009/2010) e secas (2009 e 2010) sobre a bacia dos rios Tocantins-Araguaia.

tins-Araguaia. Neste caso, observa-se que para os algoritmos com maior rBIAS (HYDROE e GSMAP), as simulações do modelo SREM2D conseguem reduzir significativamente o rBIAS, mostrando-se assim a eficácia desta ferramenta. No caso do algoritmo 3B42RT o comportamento do rBIAS em todas as escalas espaciais (tamanho de bacia) é bem próximo de zero, enquanto para o CMORPH, o conjunto das 100 realizações conseguem reduzir o rBIAS para as maiores bacias. É importante destacar que os parâmetros do modelo estocástico (SREM2D) foram calibrados para a bacia completa até a estação Tucuruí e estes mesmos parâmetros foram aplicados para cada sub-bacia a fim de verificar se um único conjunto de parâmetros poderia ser utilizado para cada uma das sub-bacias (Figura 1). A partir desta análise é possível concluir que, em todos os conjuntos de estimativa de precipitação por satélite, existe um limiar de tamanho de área (aproximadamente $12.000 \mathrm{~km}^{2}$ ) até o qual é possível observar melhoras significativas na remoção do viés utilizando os parâmetros da bacia completa. Para sub-bacias inferiores a $12.000 \mathrm{~km}^{2}$ observa-se o aumento positivo/negativo do viés, isto deve- se provavelmente a calibração do modelo SREM2D ter sido realizada para a área completa da bacia do TocantinsAraguaia, desta forma áreas com menor/maior viés são incluídas na bacia como um todo, por isso é recomendável uma calibração específica do modelo estocástico para cada uma destas sub-bacias.

\section{Conclusões}

Neste trabalho foi possível avaliar o modelo de propagação de erro SREM2D e concluir que este modelo possui potencial para gerar campos realísticos de precipitação para bacias de grande porte, complementando os estudos de Maggioni et al. (2013) e Nikolopoulos et al. (2010), aplicados a bacias de pequeno porte.

De forma geral, demonstrou-se que os algoritmos de estimativa de precipitação por satélite possuem limitações como é o caso dos algoritmos CMORPH e HYDROE que superestimam a precipitação observada. Enquanto ao GSMaP, este algoritmo subestima a precipitação observada para todo o período. Neste caso, demonstra-se como as simulações SREM2D reduziram o viés das estimativas por 


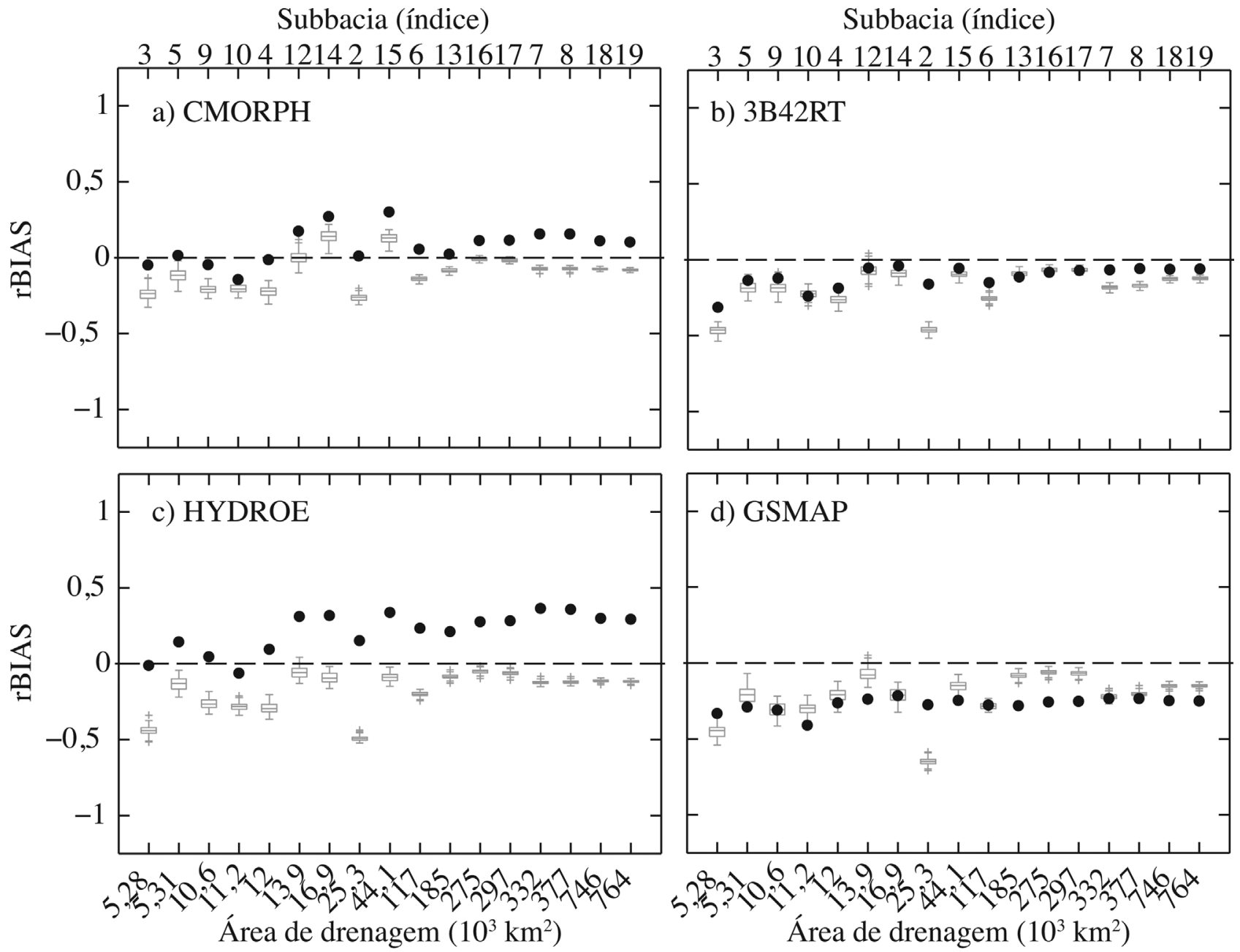

Figura 12 - Viés relativo (rBIAS) da precipitação estimada por satélite comparada com dados observados (pontos) e para um conjunto de 100 realizações (boxplot), simulado pelo SREM2D para os algoritmos CMORPH (superior-esquerdo), 3B42RT (superior-direito), HYDROE (inferior-esquerdo) e GSMaP (inferior-direito), nas estações chuvosas (2008/2009 e 2009/2010) e secas (2009 e 2010) sobre a bacia dos rios Tocantins-Araguaia.

satélite para as bacias com área superior a $12.000 \mathrm{~km}^{2}$. Desta forma, os campos de precipitação são representados com maior precisão por meio da estrutura multidimensional do erro, mostrando-se assim a eficácia do modelo estocástico SREM2D para gerar simulações de precipitação. Neste estudo foi utilizada uma única calibração para a bacia dos rios Tocantins-Araguaia (estação Tucuruí) para as estações chuvosas e secas dos anos 2008-2011. A partir da análise do viés relativo de precipitação foi possível concluir que essa calibração pode ser utilizada para diversas sub-bacias dos rios Tocantins-Araguaia levando em conta que para bacias com áreas inferiores a $12.000 \mathrm{~km}^{2}$ recomenda-se calibração específica em função da área.

Estes resultados são importantes para estudos de modelagem hidrológica a fim de avaliar a propagação do erro das estimativas de precipitação por satélite nas estimativas de vazão sobre regiões hidrográficas com baixa disponibilidade de dados. A sequência deste estudo vem sendo realizada, e será apresentada em um trabalho futuro, onde o objetivo é a avaliação da propagação das incertezas deste tipo de produto na modelagem hidrológica da bacia dos rios Tocantins-Araguaia, utilizando o Modelo Hidrológico Distribuído do Instituto Nacional de Pesquisas Espaciais (MHD-INPE), e sua aplicabilidade no monitoramento de vazões.

\section{Agradecimentos}

Esta pesquisa foi parcialmente financiada pela Coordenação de Aperfeiçoamento de Pessoal de Nível Superior (CAPES) e pelo Conselho Nacional de Desenvolvimento Científico e Tecnológico (CNPq).

\section{Referências}

ANA. Agência Nacional de Águas. Disponível em: http://hidroweb.ana.gov.br. Acesso em Janeiro de 2014.

ANAGNOSTOU, E.N., V. MAGGIONI, E. NIKOLOPOULOS, T. TAYE, F. HOSSAIN, 2010: Benchmarking High-resolution Global Satellite Rain Products to Radar and Rain gauge Rainfall Estimates, IEEE Transactions on Geo- 
sciences and Remote Sensing, Vol. 48, No 4, pp. 16671683.

AONASHI, K.; AWAKA, J.; HIROSE, M.; KOZU, T.; KUBOTA, T.; LIU, G.; SHIGE, S.; KIDA, S.; SETO, S., TAKAHASHI, N.; TAKAYABU, Y. N. GSMaP passive microwave precipitations retrieval algorithm description and validation. Journal of Meteorological Society of Japan, n. 87A, p. 119-136, 2009.

GANDIN, L. S. "On optimal interpolation and extrapolation of meteorological fields." Trudy GGO 114 (1960): 75-89.

HONG, Y.; HSU, K. L.; MORADKHANI, H.; SOROOSHIAN, S. Uncertainty quantification of satellite precipitation estimation and Monte Carlo assessment of the error propagation into hydrologic response. Water Resource Research, n. 42, W08421, p. 1-15, 2006.

HOSSAIN, F.; ANAGNOSTOU, E. N. A two-dimensional satellite rainfall error model. IEEE Transactions on Geosciences and Remote Sensing, n. 44, p. 1511-1522, 2006.

HOSSAIN, F.; HUFFMAN, G. J. Investigating Error Metrics for Satellite Rainfall Data at Hydrologically Relevant Scales. J. Hydrometeorol., n. 9, p 563-575, 2008

HOSSAIN, F.; TANG, L.; ANAGNOSTOU, E. N.; NIKOPOULOS, E. I. A Pratical guide to a space-time stochastic error model for simulation of high resolution satellite data, 2009. http://iweb.tntech.edu/fhossain/papers/SREM2DGuide.pdf.

HUFFMAN, G. J. Estimates of root mean square random error for finite samples of estimated precipitation. Journal of Applied Meteorology, vol. 36, p. 1191-1201, 1997.

HUFFMAN, G. J.; ALDER, R.; BOLVIN, D. T.; GU, G.; NELKIN, E. J.; BOWMAN, K. P.; HONG; Y. STOCKER, E. F.; WOLF, D. B. The TRMM multisatellite precipitation analysis (TMPA): Quasi-global, multiyear, combined-sensor, precipitation estimates at fine scales. J. Hydrometeorol., n. 8, p. 38-55, 2007.

IBGE/Embrapa. Instituto Brasileiro de Geografia e Estatística. Disponível em: http://mapas.ibge.gov.br/solos/viewer.htm. Acesso em Janeiro de 2014.
JOYCE, R. J.; JANOWIAK, J. E.; ARKIN, P. A.; XIE, P. CMORPH: A method that produces global precipitation estimates from passive microwave and infrared data at high spatial and temporal resolution. J. Hydrometeorol., n. 5, p. 487-503, 2004.

MAGGIONI V., H. J. Vergara, E. N. Anagnostou, J. J. Gourley, Y. Hong, and D. Stampoulis, 2013: Investigating the Applicability of Error Correction Ensembles of Satellite Rainfall Products in River Flow Simulations, J. Hydrometeorol., doi: http://dx.doi.org/10.1175/JHM-D-12-074.1

MARENGO, J. A. Interdecadal Variability and Trends of Rainfall Across the Amazon Basin. In: Theoretical a nd Applied Climatology, n. 78, p. 79-96, 2004.

MARENGO, J. A.; NOBRE, C. A. Tempo e clima no brasil. In: São Paulo: Oficina de Textos, 2009. cap. Clima da região amazônica, p. 197-212. 15

NIKOLOPOULOS, E. I., E. N. ANAGNOSTOU, F. HOSSAIN, M. GEBREMICHAEL, and M. BORGA, 2010: Understanding the scale relationships of uncertainty propagation of satellite rainfall through a distributed hydrologic model. J. Hydrometeorol., 11, 520-532.

SAPIANO, M., ARKIN, P., 2009. An intercomparison and validation of high- resolution satellite precipitation estimates with 3-hourly gauge data. J. Hydrometeorol., 10(1): 149-166.

TIAN, Y., PETERS-LIDARD, C., EYLANDER, J., JOYCE, R., HUFFMAN, G., ADLER, R., HSU, K., TURK, F., GARCIA, M., ZENG, J., 2009. Component analysis of errors in satellite-based precipitation estimates. Journal of Geophysical Research 114(D24101).

VICENTE, G.A.; SCOFIELD, R.A.; MENZEL, P.W. The operational GOES infrared rainfall estimation technique. Bulletin of the American Meteorological Society, n. 79, p. 1883-1898, 1998.

WILKS, D.S. Statistical Methods in the Atmospheric Sciences: An Introduction. Academic Press, 1995.

All the contents of this journal, except where otherwise noted, is licensed under a Creative Commons Attribution License CC-BY. 